\title{
Effect of Hydrophobic Excipients on the Release Behavior of Dexamethasone and Betamethasone from Biodegradable Poly (DL-lactide) Polymeric Implants
}

\author{
Fariba Khan, ${ }^{\mathbf{a}}$ Tasnuva Mahmud, ${ }^{\mathrm{a}}$ M. Saiful Islam, ${ }^{\mathrm{a}}$ Reza-ul Jalil ${ }^{\text {** }}$ \\ ${ }^{a}$ Department of Pharmacy, The University of Asia Pacific, Dhaka-1209; and ${ }^{b}$ Pharmaceutical \\ Technology Department, Faculty of Pharmacy, University of Dhaka, Dhaka-1000, Bangladesh.
}

\begin{abstract}
This study was conducted with the aim of formulating injectable in-situ forming implants of biodegradable poly (D,L-poly lactic acid) (DLPLA) with steroidal drugs. Two steroidal drugs betamethasone sodium phosphate and dexamethasone sodium phosphate were selected as model drug. Different hydrophobic excipients such as sorbitan esters of lauric acid (Span 20), sorbitan esters of oleic acid (Span 80), cetyl alcohol, glyceryl monostearate, glyceryl dibehenate (CompritolAT088®), glyceryl palmito-stearic ester (Precirol $\left.{ }^{\circledR}\right)$ were used. In-vitro dissolution of 7 days was performed to investigate the effect of these excipients on the release of betamethasone and dexamethasone from insitu forming DL-PLA implants. Drug release data were fitted in different models to characterize release mechanism. Both betamethasone and dexamethasone release were found to follow Korsmeyer model. Glyceryl palmitostearate was found to reduce the release of both betamethasone and dexamethasone most. Time for $25 \%$ release $\left(t_{25}\right)$, 50\% release $\left(t_{50}\right)$, and $75 \%$ release $\left(t_{75}\right)$ of the drugs were calculated. Mean dissolution time (MDT) values were also calculated from the dissolution data. MDT (day) was 2.48 \pm 0.2 and $2.31 \pm 0.3$ for betamethasone and dexamethasone respectively for implants containing no excipient. Incorporation of all the excipients (except span 20) increased these MDT values for both of the drugs indicating sustained release nature of the excipients.
\end{abstract}

Key words: In-situ implants, Poly (D,L-lactic acid), Betamethasone, Dexamethasone, Hydrophobic excipients, Sustained release

\section{Introduction}

In the past decades, several parenteral depot formulations such as implants based on biodegradable polymers have become commercially available to improve the efficacy and prolong the activity of pharmaceutical agents of a wide variety of types including, antibiotics (Koort et al., 2008), anticancer drugs (McCarron et al., 2000), steroids (Zhou et al., 1998), peptides (Rothen-Weinhold et al., 1999), proteins (Singh et al., 2001) and many other therapeutic agents.

Polymers have been used extensively in controlled drug delivery systems. There are biodegradable systems which are hydrolytically or enzymatically cleaved in vivo (Park et al., 1993) and non-degradable polymers. Biodegradation for controlling drug delivery has the advantage that the drug delivery device may not have to be removed from the site of action after drug delivery is completed and the accumulation of polymers in the body during prolonged applications is avoided (Nitsch and Banakar, 1994).

Poly(lactic acid) (PLA) and poly(D,L-lactide-co-glycolide) (PLGA) are the most frequently used biodegradable poly-

\footnotetext{
* Corresponding author: E-mail: raju1559@yahoo.com
}

mers (Anderson and Shive, 1997). Poly (lactic acid) (PLA) has been developed as a non-toxic biodegradable material which degrades in vivo to lactic acid and subsequently metabolizes to $\mathrm{CO}_{2}$ and $\mathrm{H}_{2} \mathrm{O}$ (Kulkarni et al., 1966). Yolles et al. (1971) has reported the use of this polymer for longacting injectable parenteral drug delivery systems and also obtained a patent for implants containing cyclazosin.

Glucocorticoids are used to prevent or suppress inflammation in response to multiple inciting events, including radiant, mechanical, chemical, infectious, and immunological stimuli (Hardman et al., 1996). They inhibit the production of factors that are critical in generating the inflammatory response, including vasoactive and chemoattractive factors, and lipolytic and proteolytic enzymes. In addition, they decrease the extravasion of leukocytes to the injury site (Schleimer, 1997). Betamethasone sodium phosphate and dexamethasone sodium phosphate are two glucocorticoids having high potency. They are effective on multiple organ systems also. However, they have serious systemic side effects (Liapi and Chrousos, 1992; Donald et al., 1997). 
Consequently, the controlled, continuous local delivery of these drugs at the implantation site via an in situ forming implant system has been considered as a means to avoid these side effects and achieve the goal of suppressing the local inflammatory response.

\section{Materials and Methods}

Betamethasone sodium phosphate and dexamethasone sodium phosphate were generous gift from Aristopharma Ltd. Bangladesh, D,L-poly lactic acid (DL-PLA, Boehringer Ingelheim, Ingelheim, Germany), dimethyl sulfoxide (DMSO) (Merck Limited, Mumbai, India), cetyl alcohol (BDH Chemicals Ltd., England), glyceryl monostearate (e.g. Danisco, UK), glyceryl dibehenate (e.g. Compritol AT088®, Gattefosse France), glyceryl palmitostearate (e.g. Precirol ${ }^{\circledR}$, Gattefosse France), sorbitan monolaurate (BDH Chemicals Ltd., England), sorbitan monooleate (Loba Chemie, India), zero sized capsule shell (Globe Pharmaceuticals, Bangladesh) were also used as gift.

Preparation of in-situ forming implants and In vitro dissolution study

Poly (DL-lactide) (DL-PLA) solution with $400 \mathrm{mg}$ dimethyl sulfoxide (DMSO) was prepared where rest of the ingredients (drug and excipient) were added according to Table I. Few $\mathrm{ml}$ of this solution was taken into hard gelatin capsule shell (zero sized) with the help of glass syringe (Microstat Tuberculin, USA)) and hypodermic needle (Becton Dickinson \& Company, USA). The capsule shell was immersed instantly inside a glass vials containing dissolution media of $100 \mathrm{ml}$ phosphate buffer (pH 7.4). Implants were formed in-situ in the dissolution media. Dissolution vessels (glass vials) containing the dissolution media were kept in a thermostatic water bath (Memmert GmbH \& Co.
KG, Germany) maintaining the rpm at 50 and temperature at $37 \pm 0.5^{\circ} \mathrm{C}$. Dissolution samples were then withdrawn from each of the vials at predetermined intervals. This study was carried out for seven days. Dissolution samples were analyzed spectrophotometrically at $241 \mathrm{~nm}$ (betamethasone) and $241.5 \mathrm{~nm}$ (dexamethasone) (BP 2003). This whole procedure was performed in triplicate.

\section{Kinetic Analysis of Dissolution Data}

To study the mechanism of drug release from the implants, the release data were fitted to zero-order, first-order, and Higuchi equation (Higuchi, 1963). These models fail to explain drug release mechanism. Therefore, the dissolution data was also fitted to the well-known exponential equation (Korsmeyer equation), which is often used to describe the drug release behavior from polymeric systems (Korsmeyer et al., 1983).

$\log \left(\mathrm{M}_{\mathrm{t}} / \mathrm{M}_{\mathrm{f}}\right)=\log \mathrm{k}+\mathrm{n} \log \mathrm{t}$

where, Mt is the amount of drug release at time t; $\mathrm{Mf}$ is the amount of drug release after infinite time; $\mathrm{k}$ is a release rate constant incorporating structural and geometric characteristics of the implant; and $\mathrm{n}$ is the diffusional exponent indicative of the mechanism of drug release.

To clarify the release exponent for different batches of sustained release implants, the log value of percentage drug dissolved was plotted against log time for each batch according to the equation 1. A value of $\mathrm{n}<0.45$ indicates Fickian (case I) release; $>0.45$ but $<0.89$ for non-Fickian (anomalous) release; and $>0.89$ indicates super case II type of release. Case I generally refers to diffusion controlled drug release, Case II refers to the erosion of the polymeric chain and anomalous transport (Non-Fickian) refers to a combination

Table I: Composition of Sustained release Biodegradable Implants of Betamethasone/ Dexamethasone sodium phosphate (20 mg)

\begin{tabular}{l|c|c|c|c|c|c|c}
\hline Batch & $\begin{array}{c}\text { DL- } \\
\text { PLA (mg) }\end{array}$ & $\begin{array}{c}\text { Cetyl } \\
\text { Alcohol (mg) }\end{array}$ & $\begin{array}{c}\text { Glyceryl } \\
\text { Dibehenate (mg) }\end{array}$ & $\begin{array}{c}\text { Glyceryl } \\
\text { Palmitostearate (mg) }\end{array}$ & $\begin{array}{c}\text { Glyceryl } \\
\text { Monostearate (mg) }\end{array}$ & $\begin{array}{c}\text { Span } \\
20 \text { (mg) }\end{array}$ & $\begin{array}{c}\text { Span } \\
80 \text { (mg) }\end{array}$ \\
\hline A & 200 & 20 & & & & & \\
B & 200 & & 20 & 20 & 20 & & \\
C & 200 & & & & & 20 & 20 \\
D & 200 & & & & & \\
E & 200 & & & & & \\
F & 200 & & & & & \\
\hline Blank & 200 & & \multicolumn{5}{c}{ No excipient } \\
\hline
\end{tabular}


of both diffusion and erosion controlled drug release (Peppas, 1985).

Mean dissolution time (MDT) was calculated from dissolution data using the following equation (Mockel and Lippold, 1993):

$\operatorname{MDT}=(\mathrm{n} / \mathrm{n}+1) \cdot \mathrm{K}^{-1 / \mathrm{n}}$

Where, $\mathrm{n}$ = release exponent and $\mathrm{k}=$ release rate constant.

\section{Results and Discussion}

DL-PLA implants of betamethasone and dexamethasone were formed in-situ (Fig.1) in the dissolution media. Dissolution study was conducted for 7 days.

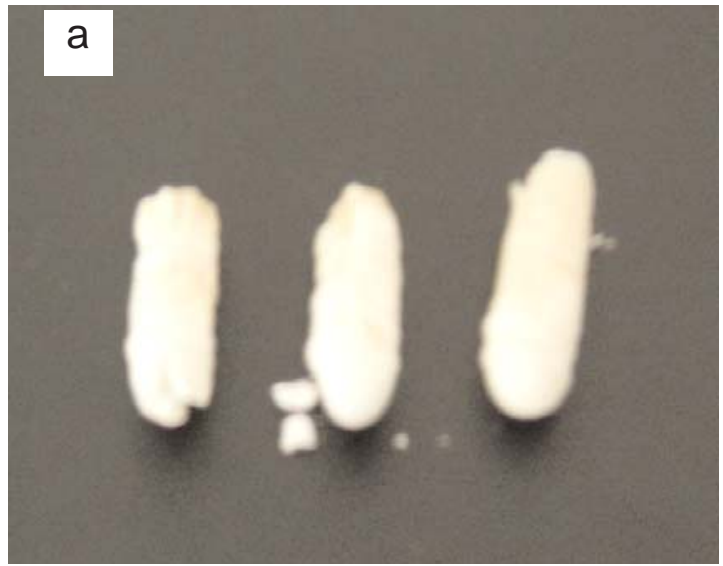

Fig.1. Physical morphology of in-situ forming biodegradable DL-PLA implants of betamethasone (a) and dexamethasone (b)

Figure 2 shows the in vitro release of betamethasone from DL-PLA implants. Implants containing no excipient (blank) released $76.12 \pm 2.01 \%$ betamethasone in $\mathrm{pH} 7.4$ buffer media after 7-days. Cetyl alcohol (CA) (batch A) caused a little sustained release of the drug. It was $73.52 \pm 2.014 \%$ while CA was incorporated into the implant. CA is an emulsifying and stiffening agent. It occurs as waxy, white flake which is practically insoluble in water (Kibbe, 2000). This water insoluble nature of the CA might play a role in retarding the release of the betamethasone slightly. Moreover, CA is also used as sustained release excipient in extending the release of the drug (Nguyen et al., 2008; Castellanos et al., 2008). Batch B, C, D containing glyceryl dibehenate, glyceryl palmitostearate, and glyceryl monostearate respectively also reduced the release of betamethasone but in a greater extent (Fig. 2). Glyceryl dibehenate (GB) reduced the release of the drug to $72.14 \pm 3.124 \%$. GB is a tablet and capsule

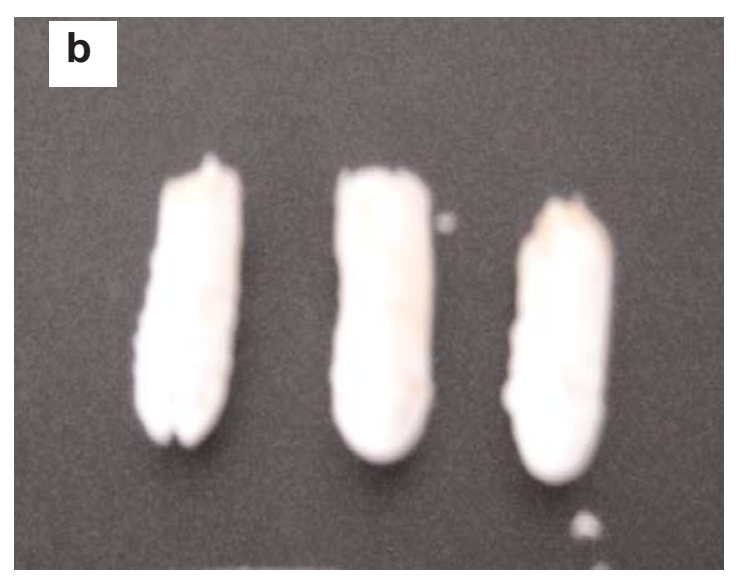

lubricant (Abramovici et al., 1986). It is a mixture of glycerides of fatty acids, mainly behenic acid and is also practically insoluble in water (Kibbe, 2000). This lipophilic nature might hinder the release of the water soluble betamethasone. But most importantly, GB has a good release retarding capacity which played the major role in controlling the release of betamethasone from DL-PLA implants (Li et al., 2006; Obaidat and Obaidat, 2001; Perez et al., 1993). Similar but more pronounced reduced release was observed while glyceryl monostearate (GMS) was incorporated in the implant formulation (batch D). Percent release of betamethasone was $67.45 \pm 4.014 \%$ for this batch. GMS is a lipophilic emulsifying agent (HLB 3.8) which is practically insoluble in water (Kibbe 2000). Besides, it is a lubricant for tablet

manufacturing and may be used to form sustained release matrices for solid dosage forms (Peh and Yuen, 1995). However, GMS retarded the release of betamethasone from in-situ forming implants in this experiment where implants were made using biodegradable DL-PLA polymer. This release retardant activity of GMS from biodegradable implantable dosage form was also evident previously (Peri et al., 1994). Amongst the excipients, glyceryl palmitostearate (GPS) was able to reduce the release of betamethasone most. After incorporation of this excipient, betamethasone release was $58.21 \pm 2.214 \%$ after 7 days while this figure was 76.12 $\pm 2.01 \%$ for the blank (Fig. 2b). GPS is also a water insoluble excipient which is used as tablet and capsule lubricant. It is a mixture of mono-, di-, and tri-, glycerides of C16 and C18 fatty acids (Kibbe 2000). Due to this more lipophilic nature of the GPS, it has been used as a matrix former in sustained release dosage forms (Shaikh et al., 1991). Gao et al. 

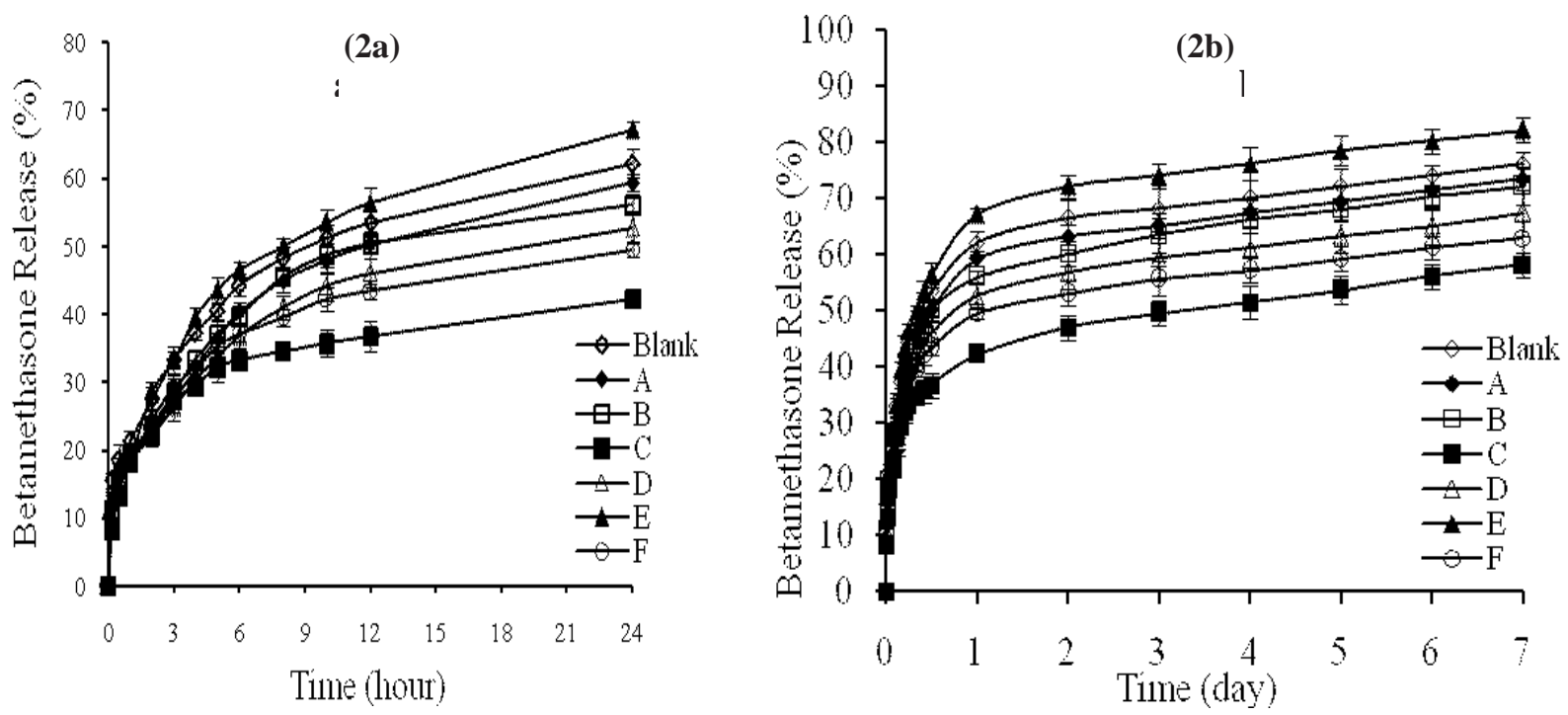

Fig. 2. In vitro release profiles of betamethasone from biodegradable DL-PLA implants after 1 day (a) and 7-day (b) of dissolution where blank=no excipient, $\mathrm{A}=$ cetyl alcohol, $\mathrm{B}=$ glyceryl dibehenate, $\mathrm{C}=$ glyceryl palmitostearate, $\mathrm{D}=$ glyceryl monostearate, $\mathrm{E}=$ span 20 and $\mathrm{F}=$ span 80.Data are represenred as mean $\pm \mathrm{SD}(\mathrm{n}=3)$

(1995) also showed that GPS could be a good release retardant for steroids while it was used in a biodegradable injectable dosage form.

Sorbitan monolaurate (Span 20) and sorbitan monooleate (Span 80) were also used in the betamethasone implant formation (Table I). Batch E containing span 20 increased the release of the drug $(82.14 \pm 2.214)$ which is shown in Figure 2. It might be due to less lipophilic nature (HLB 8.6) of span 20 (Kibbe 2000). On the other hand, span 80 (batch F) showed more release controlling capacity of betamethasone compared with span 20 (Fig. 2). Betamethasone release was $62.89 \pm 3.12 \%$ for this batch. This is attributable to the fact that presence of hydrophobic excipient, span 80, in the matrix impedes the rate of entrance of dissolution medium within the matrix and hence the dissolution of water soluble
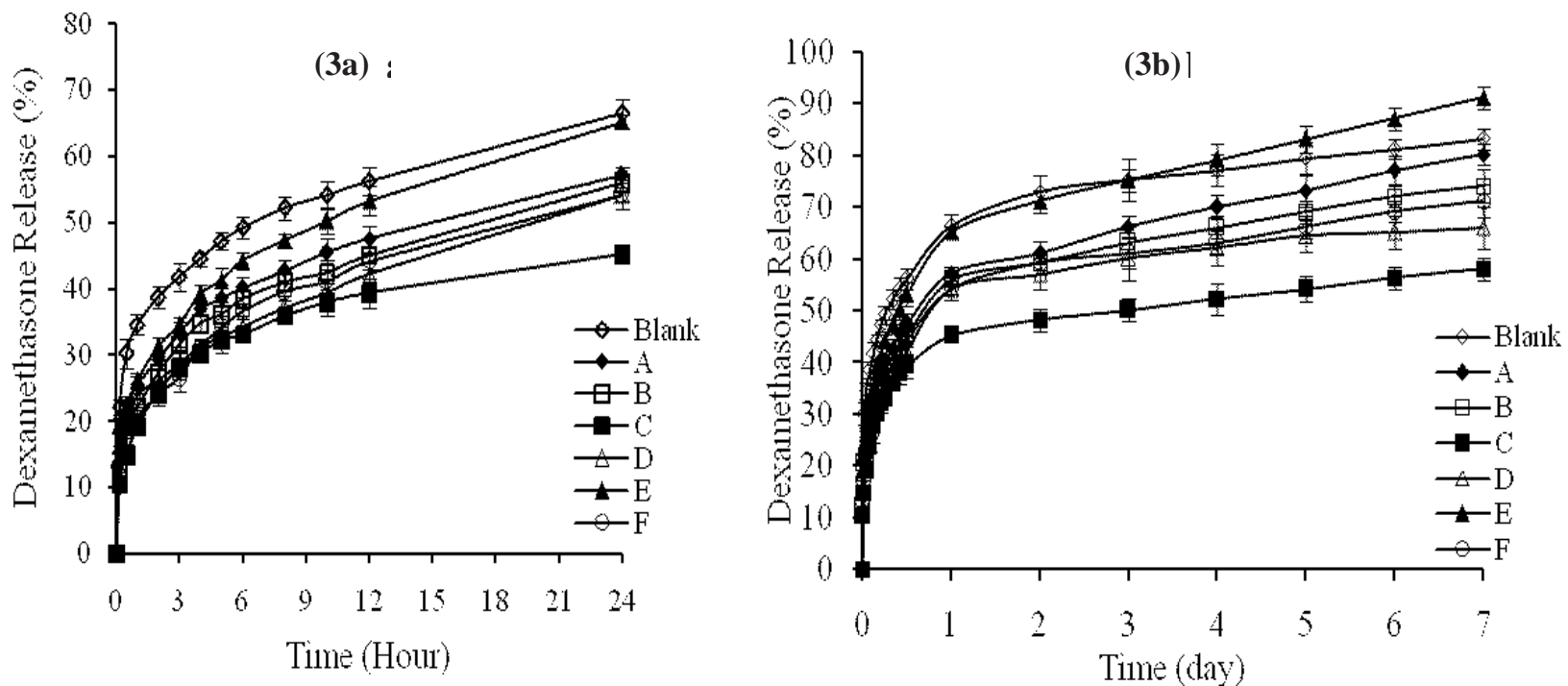

Fig. 3. In vitro release profiles of dexamethasone from biodegradable DL-PLA implants after 1 day (a) and 7-day (b) of dissolution where blank=no excipient, $\mathrm{A}=$ cetyl alcohol, $\mathrm{B}=$ glyceryl dibehenate, $\mathrm{C}=$ glyceryl palmitostearate, $\mathrm{D}=$ glyceryl monostearate, $\mathrm{E}=$ span 20 and $\mathrm{F}=$ span 80.Data are represenred as mean $\pm \mathrm{SD}(\mathrm{n}=3)$ 
betamethasone was slowed considerably. The hydrophobic nature of surfactant, span 80 is due to its very low HLB value of 4.3 (Kibbe, 2000).

These excipients, except span 20, were also able to control the burst release of water soluble betamethasone sodium phosphate from DL-PLA implants as shown in Figure 2a. In the figure, release data of betamethasone after 1 (one) day is shown.

Biodegradable DL-PLA implants were also made with dexamethasone sodium phosphate, another potent glucocorticoids having a comparative glucocorticoid potency of 2580(Liapi and Chrousos, 1992; Donald et al., 1997). Similar release patterns, as were observed in case of betamethasone, were also observed for dexamethasone for individual excipient (Fig. 3a). Dexamethasone release after 7-days of dissolution was $83.25 \pm 2.01 \%$ from implants formulated without excipient (blank). After the incorporation of CA (batch A), GB (batch B), GPS (batch C), GMS (batch D), span 20 (batch E) and span 80 (batch F), dexamethasone release was 80.21 $\pm 2.01 \%$, 74.12 $\pm 3.12 \%$, 58.12 $\pm 2.21 \%, 66.12 \pm 4.01 \%$, $91.21 \pm 2.21 \%$, and $71.21 \pm 3.12 \%$ respectively (Fig. 3b). Incorporation of these excipients also reduced dexamethasone release markedly after 1 (one) day of dissolution (Fig. 3a).

The release rate kinetic data for all the models is shown in Table II. Release data for betamethasone and dexamethasone of all the batches were fitted in Korsmeyer equation. Regression co-efficient $\left(r^{2}\right)$ values ranged from 0.934 to 0.959 for betamethasone and 0.948 to 0.99 for dexamethasone and indicated diffusion controlled release mechanisms of the drugs. Value of release exponent " $n$ " determined from the various formulations ranged from 0.24 to 0.272 for betamethasone and 0.185 to 0.247 for dexamethasone (Table II). The $\log \mathrm{k}$ values are also shown in Table II where the values ranged from 0.241 to 0.396 for betamethasone and 0.21 to 0.383 for dexamethasone. The value of " $n$ " and log $\mathrm{k}$ was found to vary with type of excipients in the DL-PLA implants.

The time taken to release $25 \%\left(t_{25}\right), 50 \%\left(t_{50}\right)$, and 75\% $\left(t_{75}\right)$ of betamethasone and dexamethasone from DL-PLA implants formulated with different excipients was determined from the dissolution data (Table III). DL-PLA implants of betamethasone containing no excipient (blank) required $0.04 \pm 0.01$ and $3.9 \pm 0.2$ days to release $25 \%$ and $75 \%$ of drug respectively. All the excipients increased these values for betamethasone except span 20 (batch E). Amongst the excipients, glyceryl palmitostearate caused maximum time to release $25 \%(0.15 \pm 0.02$ day) and $75 \%(12.14 \pm 0.2$ day) of betamethasone. These excipients also increased $t_{25}$, $\mathrm{t} 50$, and $\mathrm{t}_{75}$ of dexamethasone from DL-PLA implants (see Table III). $t_{25}$, and $t_{75}$ values for dexamethasone were $0.11 \pm 0.01$ and $13.4 \pm 0.5$ day respectively as glyceryl palmitostearate was incorporated in the implants. Where as these

Table II: Mathematical Modeling and Drug Release Kinetics of Betamethasone and Dexamethasone Implants

\begin{tabular}{l|c|c|c|c|c|c|c}
\hline \multirow{2}{*}{ Batch } & & \multicolumn{3}{|c|}{$\mathrm{r}^{2}$ values } & & \\
\cline { 3 - 8 } & & Zero Order & First Order & Higuchi & Korsmeyer Model & LogK & $\mathrm{n}$ \\
\hline Betamethasone & $\mathrm{A}$ & 0.625 & 0.767 & 0.819 & 0.95 & 0.295 & 0.264 \\
Sodium Phosphate & $\mathrm{B}$ & 0.62 & 0.764 & 0.812 & 0.942 & 0.302 & 0.267 \\
& $\mathrm{C}$ & 0.643 & 0.755 & 0.823 & 0.934 & 0.396 & 0.25 \\
& $\mathrm{D}$ & 0.629 & 0.753 & 0.821 & 0.959 & 0.335 & 0.268 \\
& $\mathrm{E}$ & 0.61 & 0.792 & 0.807 & 0.936 & 0.241 & 0.272 \\
& $\mathrm{~F}$ & 0.604 & 0.723 & 0.797 & 0.938 & 0.351 & 0.25 \\
Dexamethasone & Blank & 0.603 & 0.76 & 0.799 & 0.948 & 0.266 & 0.24 \\
Sodium Phosphate & $\mathrm{A}$ & 0.718 & 0.885 & 0.881 & 0.989 & 0.275 & 0.227 \\
& $\mathrm{~B}$ & 0.688 & 0.837 & 0.863 & 0.975 & 0.3 & 0.24 \\
& $\mathrm{C}$ & 0.615 & 0.725 & 0.802 & 0.948 & 0.383 & 0.229 \\
& $\mathrm{D}$ & 0.662 & 0.776 & 0.848 & 0.968 & 0.332 & 0.24 \\
& $\mathrm{E}$ & 0.729 & 0.931 & 0.893 & 0.99 & 0.228 & 0.236 \\
& F & 0.687 & 0.817 & 0.866 & 0.98 & 0.322 & 0.247 \\
\hline
\end{tabular}


Table III. Dissolution Parameters of Betamethasone and Dexamethasone from DL-PLA implants

\begin{tabular}{l|c|c|c|c|c}
\hline \multicolumn{2}{l|}{ Batch } & t25\% (day) & t50\% (day) & t75\% (day) & MDT (day) \\
\hline Betamethasone & A & $0.07 \pm 0.01$ & $0.95 \pm 0.1$ & $4.4 \pm 0.1$ & $2.73 \pm 0.2$ \\
sodium phosphate & B & $0.075 \pm 0.01$ & $1.01 \pm 0.2$ & $4.6 \pm 0.2$ & $2.85 \pm 0.2$ \\
& C & $0.15 \pm 0.02$ & $2.4 \pm 0.1$ & $12.14 \pm 0.2$ & $7.68 \pm 0.5$ \\
& D & $0.1 \pm 0.01$ & $1.33 \pm 0.07$ & $6.07 \pm 0.5$ & $3.76 \pm 0.1$ \\
& E & $0.05 \pm 0.02$ & $0.6 \pm 0.03$ & $2.67 \pm 0.3$ & $1.65 \pm 0.1$ \\
& F & $0.1 \pm 0.01$ & $1.6 \pm 0.1$ & $8.02 \pm 0.4$ & $5.08 \pm 0.2$ \\
Dexamethasone & Blank & $0.04 \pm 0.01$ & $0.71 \pm 0.1$ & $3.9 \pm 0.2$ & $2.48 \pm 0.2$ \\
sodium phosphate & A & $0.036 \pm 0.01$ & $0.76 \pm 0.1$ & $4.6 \pm 0.2$ & $3.01 \pm 0.2$ \\
& B & $0.055 \pm 0.03$ & $0.99 \pm 0.3$ & $5.4 \pm 0.3$ & $3.44 \pm 0.3$ \\
& C & $0.11 \pm 0.01$ & $2.28 \pm 0.08$ & $13.4 \pm 0.5$ & $8.76 \pm 0.4$ \\
& D & $0.074 \pm 0.02$ & $1.34 \pm 0.1$ & $7.3 \pm 0.4$ & $4.67 \pm 0.3$ \\
& E & $0.026 \pm 0.01$ & $0.5 \pm 0.1$ & $2.7 \pm 0.2$ & $1.76 \pm 0.1$ \\
& F & $0.073 \pm 0.01$ & $1.22 \pm 0.2$ & $6.3 \pm 0.3$ & $3.98 \pm 0.3$ \\
& Blank & $0.007 \pm 0.01$ & $0.32 \pm 0.1$ & $2.9 \pm 0.1$ & $2.13 \pm 0.3$ \\
\hline
\end{tabular}

values were only $0.07 \pm 0.01$ and $2.9 \pm 0.1$ day for implants containing no excipient (blank).

Mean dissolution time (MDT) value is used to characterize drug release rate from a dosage form and indicates the drug release retarding efficiency of polymer. MDT values for betamethasone and dexamethasone were also calculated from the dissolution data (Table III). As shown in the table, MDT values of the implants formulated without excipient were $2.48 \pm 0.2$ and $2.13 \pm 0.3$ day for betamethasone and dexamethasone respectively. Except span 20, all the excipients increased this MDT values. But implants prepared with glyceryl palmitostearate showed the highest MDT values. It was $7.68 \pm 0.5$ day for betamethasone and $8.76 \pm 0.4$ day for dexamethasone.

\section{Conclusion}

Results of the present study demonstrated that different types of lipophilic surfactants and lubricants/diluents could be successfully employed for formulating sustained release biodegradable DL-PLA implants for betamethasone and dexamethasone. Incorporation of the excipients resulted differences in release behavior, especially the burst effect, which is a very crucial point in efficacy and safety of in-situ forming injectable implant. This can be expected to reduce the frequency of administration and decrease the dosedependent side effects associated with repeated administration of conventional betamethasone and dexamethasone tablets.

\section{Acknowledgments}

Authors are grateful to Aristopharma Ltd., Bangladesh for providing betamethasone sodium phosphate and dexamethasone sodium phosphate as gift sample.

\section{References}

Abramovici B., Gromenil J. C., Molard F. and Blanc F. (1986) Comparative study of the lubricant properties of a new excipient, Glyceryl tribehenate (Compritol 888) compared with magnesium stearate. STP Pharma. 2: 403-409.

Anderson J. M. and Shive M. S. (1997) Biodegradation and biocompatibility of PLA and PLGA microspheres, Adv. Drug Deliv. Rev. 28: 5 - 27.

British Pharmacopoeia, 2003.

Castellanos Gil E., Iraizoz Colarte A., Lara Sampedro J. L. and Bataille B. (2008) Subcoating with Kollidon VA 64 as water barrier in a new combined native dextran/HPMC-cetyl alcohol controlled release tablet. Eur J Pharm Biopharm. 69(1): 303-11.

Gao Z. H., Shukla A. J., Johnson J. R. and Crowley W. R. (1995) Controlled release of contraceptive steroids from biodegradable and injectable gel: in vivo evaluation. Pharm Res. 12: 864-868. 
Hardman J., Limbird L., Molinoff P., Ruddon R. and Gliman A. (1996) Goodman and Gilman's the pharmacological basis of therapeutics. 9th Ed. (New York: McGrawHill).

Higuchi T. (1963) Mechanism of sustaineded-action medication: theoretical analysis of rate of release of solid drugs dispersed in solid matrices. J Pharm Sci. 52: 1145-1149.

Kibbe A. H. (2000) Handbook of Pharmaceutical Excipients. 3rd Ed. (Washington, D.C.: USA: American Pharmaceutical Association).

Koort J. K., Makinen T. J., Suokas E., Veiranto M., Jalava J., Tormala P. and Aro, H. T. (2008) Sustained release of ciprofloxacin from an osteoconductive poly(DL)-lactide implant, Acta Orthop. 79(2): 295-301.

Korsmeyer R.W., Gurny R., Docler E., Buri P. and Peppas N. A. (1983) Mechanism of solute release from porous hydrophilic polymers. Int J Pharm. 15:25-35.

Kulkarni R. K., Pani K. C., Neuman C. and Leonard F. (1966) Polylactic acid for surgical implants. Arch. Surg. 93: 839-843.

Leung Y. M., Hanifin J. M. and Charlesworth E. N. (1997) Disease management of atopic dermatitis: a practice parameter; Annals of allergy, asthma, \& immunology; volume 79.

Li F. Q., Hu J. H., Deng J. X., Su H., Xu S. and Liu J. Y. (2006) In vitro controlled release of sodium ferulate from Compritol 888 ATO-based matrix tablets. Int $J$ Pharm. 324(2): 152-7.

Liapi C. and Chrousos G. P. (1992) Glucocorticoids. In: Jaffe SJ, Aranda JV (eds). Pediatric Pharmacology. 2nd Ed. (WB Saunders Co, Philadelphia), pp 466-475.

McCarron P. A., Woolfson A. D. and Keating, S. M. (2000) Sustaineded release of 5-fluorouracil from polymeric nanoparticles. J. Pharm. Pharmacol. 52: 1451-1459.

Mockel J. E. and Lippold B. C. (1993) Zero order release from hydrocolloid matrices. Pharm Res. 10: 10661070 .
Nguyen C. N., Christensen J. M. and Ayres J. W. (2008) Formulation of sustaineded-release verapamil $\mathrm{HCl}$ and diltiazem $\mathrm{HCl}$ semisolid matrix capsules. Pharm Dev Technol. 13(2): 115-25.

Nitsch M. J. and Banakar U. V. (1994) Implantable drug delivery, in Advances in controlled delivery of drugs. Technomic Publishing Company, I, Editor. (Technomic Publishing Company, Inc.Tech: Lancaster), pp. 21 - 58.

Obaidat A. A. and Obaidat R. M. (2001) Controlled release of tramadol hydrochloride from matrices prepared using glyceryl behenate. Eur J Pharm Biopharm. 52(2): 231-235.

Park K., Shalaby W. S. W. and Park H. (1993) Biodegradation, in Biodegradable hydrogels for drug delivery, Technomic publishing company, I, Editor. (Technomic Publishing Company, Inc: Lancaster), pp. 13 - 34.

Peh K. K. and Yuen K. H. (1995) Development and in vitro evaluation of a novel multiparticulate matrix controlled release formulation of theophylline. Drug Dev Ind Pharm. 21: 1545-1555.

Peppas N. A. (1985) Analysis of Fickian and non-Fickian drug release from polymers. Pharm Acta Helv. 60: 110-111.

Perez M. A., Ghaly E. S. and Marti A. (1993) Sustaineded release phenylpropanolamine hydrochloride from ATO 888 matrix. P R Health Sci J. 12(4): 263-267.

Peri D., Bogdansky S., Allababidi S. and Shah J. C. (1994) Development of an implantable, biodegradable, controlled drug delivery system for local antibiotic therapy. Drug Dev Ind Pharm. 20: 1341-1352.

Rothen-Weinhold A., Besseghir K., Vuaridel E., Sublet E., Oudry N. and Gurny, R. (1999) Stability studies of a somatostatin analogue in biodegradable implants. Int. J. Pharm.178: 213-221.

Schleimer R., Busse W. and O'Byrne P. (1997) Inhaled glucocorticoids in asthma. In: Lenfant $\mathrm{C}$, editor. Lung biology. Health and disease, (New York: Marcel Dekker), 97: 773. 
Shaikh N. H., De Yanes S. E., Shukla A. J., Block L. H., Collins C. C. and Price J. C. Effect of different binders on release characteristics of theophylline from compressed microspheres. Drug Dev Ind Pharm. 17: 793804.

Singh M., Shirley B., Bajwa K., Samara E., Hora M. and O'Hagan D. (2001) Controlled release of recombinant insulin-like growth factor from a novel formulation of polyactide-co-glycolide microparticles. J. Control Rel. 70: $21-28$.
Yolles S., Eldridge J. E. and Woodland J. H. R. (1971) New biodegradable drug delivery systems. Polymer News. 1: 9.

Zhou T., Lewis H., Foster R. E. and Schwendeman S.P. (1998) Development of a multiple-drug deliveryimplant for intraocular management of proliferative vitreoretinopathy. J. Control Rel. 55: 281-/295.

Received : May 13, 2009;

Accepted : January 06, 2010 